\title{
Effect of platelet-rich plasma combined with demineralised bone matrix on bone healing in rabbit ulnar defects
}

\author{
Vasilios Galanis ${ }^{1}, \mathrm{MD}$, Alice $\underline{\text { Fiska }}{ }^{2}, \mathrm{MD}, \mathrm{PhD}$, Stylianos ${\underline{K a p e t a n a k i s^{2}}}, \mathrm{MD}, \mathrm{PhD}$, Konstantinos $\underline{K a z a k o s}^{1}, \mathrm{MD}, \mathrm{PhD}$,
} Thespis Demetriou ${ }^{2}$, MD, PhD

\begin{abstract}
INTRODUCTION This study evaluates the effect of autologous platelet-rich plasma (PRP) combined with xenogeneic demineralised bone matrix (DBM) on bone healing of critical-size ulnar defects (2-2.5 times the ulnar diameter) in New Zealand White rabbits.

METHODS Critical-size defects were created unilaterally in the ulna of 36 rabbits, while keeping the contralateral limb intact. They were divided into three groups. In Group A, the defect was filled with autologous PRP and in Group B, with autologous PRP combined with DBM; in Group C, the defect remained empty. The rabbits were euthanised 12 weeks postoperatively. Radiological, biomechanical and histological assessments were carried out and statistical analysis of the results was performed.

RESULTS Group B had significantly higher radiological and histological scores than Groups A and C. Defects in Group B showed significant new bone formation, whereas there was minimal or no new bone formation in Groups A and C. Only specimens in Group B showed macroscopic bone union. Biomechanical evaluation of the treated and intact contralateral limbs in Group B showed significant differences.

CONCLUSION In this study, statistically significant enhancement of bone healing was found in critical-size defects treated with PRP and DBM, as shown by radiological findings, gross assessment, and biomechanical and histopathological results. Defects in the two other groups remained unbridged. Therefore, PRP was effective only when it was used in combination with a bone graft.
\end{abstract}

Keywords: demineralised matrix, platelet-rich plasma, union

\section{INTRODUCTION}

Bone grafting procedures can promote the healing of criticalsize osseous defects. The autologous bone graft remains the gold standard of treatment for delayed union or non-union of long bone fractures. The risk of donor morbidity is present, but can be avoided by the application of bone graft substitutes with osteoconductive and/or osteoinductive properties in various combinations. ${ }^{(1,2)}$ In current practice, orthopaedic surgeons mainly use demineralised bone matrix (DBM) as an osteoconductive material that has some osteoinductive properties to induce bone formation. ${ }^{(3)}$

Platelet-rich plasma (PRP) is increasingly used in many surgical fields and has various soft tissue applications, such as in chronic tendinosis and the treatment of chronic, difficult wounds, with good results. ${ }^{(4,5)} \mathrm{PRP}$ is an autologous blood-derived product with an increased concentration of platelets. It is rich in numerous growth factors that promote the recruitment, proliferation and differentiation of cells involved in tissue regeneration. Thus, PRP has the potential to enhance tissue healing at the cellular level. These growth factors can also stimulate the proliferation and differentiation of cells of osteogenic lineage and, therefore, PRP may be used to promote bone healing. ${ }^{(6)}$ In the literature, there are few experimental studies on the use of PRP in combination with DBM to enhance bone union in large bone defects. ${ }^{(7,8)}$ The present study aimed to determine if autologous PRP (prepared from rabbit blood) alone or used in combination with xenogeneic DBM promotes osteogenesis when applied to critical-size defects in rabbit ulnas.

\section{METHODS}

A total of 36 New Zealand White rabbits were used in this study, with an average weight of 3.5 (range 3.3-3.7) kg. Approval from the regional ethical board was obtained (Animal Health Service T/2174/25-6-2009). All guidelines for the care and treatment of laboratory animals were adhered to. Randomisation of the animals was performed without considering their sex. All the rabbits were skeletally mature; preoperative radiological evaluation was done to ensure skeletal maturity and the absence of abnormalities. The rabbits were singly housed with food and water available ad libitum.

A unilateral, critical-size ulnar defect was surgically created in each rabbit, while the contralateral limb remained intact. They were subdivided into three groups of 12 rabbits each and the ulnar defect was treated with PRP, treated with PRP combined with DBM or not treated. Group A received only PRP in the critical-size defect. Group B received PRP in combination with xenogeneic (human) DBM. Human DBM was used with carrier collagen (Accell, IsoTis OrthoBiologics, Irvine, CA). In Group C,

${ }^{1}$ Department of Orthopaedics, University Hospital of Alexandroupolis, ${ }^{2}$ Department of Anatomy, Medical School, Democritus University of Thrace, Greece Correspondence: Dr Stylianos Kapetanakis, Orthopaedic Surgeon, Assistant Professor of Anatomy, Department of Anatomy, Medical School, Democritus University of Thrace, Dragana, Alexandroupolis 68100, Greece. stkapetanakis@yahoo.gr 
a simple closure of surrounding soft tissue was performed in the unilateral ulnar defect, with no material in the defect. All the animals were evaluated using radiography of the forelimbs immediately after the operation as well as at postoperative weeks 4 and 12 . At the end of the study period, 12 weeks postoperatively, the animals were euthanised and sent for histological study and biomechanical testing.

PRP was prepared using PRP Fast (Bioteck, Vicenza, Italy). Before the surgical procedure, two animals from each group were used as donors. Their platelet counts were measured with a standard haemocytometer, and concentrations of transforming growth factor beta-1 (TGFB1) and platelet-derived growth factorBB (PDGF-BB) of blood and the extracted PRP were taken with enzyme-linked immunosorbent assay kits, according to the manufacturer's instructions. After anaesthesia and prior to the surgical procedure, $8 \mathrm{~mL}$ of autologous blood was collected from an ear vein and $2 \mathrm{~mL}$ of PRP was prepared, as per the manufacturer's instructions, from each rabbit. Blood collected in a tube was allowed to stand for 15 minutes in order to reduce platelet activation during centrifugation. Following centrifugation, the upper half of the suspension, consisting of platelet-poor plasma, was removed using sterile pipettes. The lower half (i.e. PRP) was selected with a pipette by aspirating it up to the interface zone, consisting of blood cells. For every $8 \mathrm{~mL}$ of blood, about $2 \mathrm{~mL}$ of PRP was produced. According to the manufacturer's instructions, the PRP produced had 3-7 times more platelets and growth factors after centrifugation than blood. In this study, PRP was used in a gel form after adding calcium chloride solution in a ratio of 10:1 for secure application to the osseous defect alone, or mixed well with DBM.

Intramuscular injections of xylazine (Rompun ${ }^{\mathrm{TM}}$ Injectable; Bayer HealthCare, Shawnee, Kansas, USA) 5-7 mg/kg, atropine (DEMO, Krioneri, Attica, Greece) $0.15 \mathrm{mg}$ and ketamine (Imalgene; Rhone Merieux, Lyon, France) 12-15 mg/kg were used for the rabbits' anaesthesia. Supplemental sedation was given during surgery when required. At regular intervals, $1 \mathrm{~mL}$ $2 \%$ lidocaine-adrenaline solution (AstraZeneca, London, United Kingdom) was injected at the incision site as local anaesthesia. The forelimbs of the rabbits were then shaved. Using standard aseptic techniques, a lateral ulnar approach was made at the border of the upper- and middle-third of the ulna unilaterally, where the bone is subcutaneous. After dissection of the periosteum, bony defects were created with an oscillating saw. The size of the defect was approximately 2-2.5 times the ulna diameter in this area, as measured intraoperatively with a ruler. This skeletal gap represented a critical-size defect that would not heal spontaneously, according to the literature. ${ }^{(9-11)}$ Radiography performed immediately after the operation also confirmed that the defects were of the required size. The defects were irrigated with saline solution to remove bone debris.

The graft materials were carefully placed in the osseous defects of the rabbits in the two treatment groups. DBM was mixed with autologous PRP before administration to Group B defects. The periosteum and the surrounding soft tissues were enclosed in layers with running sutures, forming an envelope to hold the filling of the osseous defect. The radius provided mechanical stability and internal fixation was not used. The skin was closed with clips. Immobilisation was not required after surgery, and the rabbits were allowed to perform their daily activities in their cages without restriction. They were treated with intramuscular antibiotics for three days after the operation and analgesics, as required.

At the end of the study period, 12 weeks postoperatively, the animals were sacrificed with a $10 \%$ potassium chloride intracardiac injection under general anaesthesia. The ulnas and radii were harvested and placed in saline solution to be prepared for biomechanical testing and histological assessment. Anteroposterior radiographs of the forelimbs were obtained immediately after surgery as well as at postoperative weeks 4 and 12 . The radiographs were evaluated using the six-point radiographic grading scale for the degree of healing defined by Cook et al. ${ }^{(12)}$ Radiological scores in this system range from a minimum of 0 (no change from immediate postoperative appearance) to a maximum of 6 (defects absolutely bridged with new bone).

After the rabbits were sacrificed, the specimens were surgically prepared. Soft tissue was removed from the defect site together with contralateral tissue. One-third of the specimens were delivered to the laboratory for biomechanical testing according to a protocol similar to that of Karachalios et al. ${ }^{(13)} \mathrm{A}$ gross assessment was performed macroscopically and specimens were tested only when their callus was found to be stable. For biomechanical evaluation, a computerised testing machine (Imperial 2500, N Load Cell ILC 2500N; Mecmesin, Horsham District, West Sussex, UK) was used. A digital caliper (500-181U; Mitutoyo, Kawasaki, Kanagawa, Japan) and a digital photo and video recorder (DCR-TRV80E; Sony, Minato, Tokyo, Japan) were used to capture still pictures and videos during biomechanical testing. Biomechanical testing of specimens involved loading under a three-point bending configuration. The two lower supports were fixed at a centre-to-centre distance of $30 \mathrm{~mm}$ and the upper support was driven downwards at a speed of $0.05 \mathrm{~mm} / \mathrm{s}$ or $3 \mathrm{~mm} / \mathrm{min} .{ }^{(14)}$

The treated specimens were placed on the two lower supports with the mid-callus region along the loading axis and the concave diaphyseal aspect facing upwards. The control specimens were similarly placed on the two lower supports with their corresponding site along the loading axis. During loading, force and displacement were recorded at a sampling frequency of $100 \mathrm{~Hz}$, and curves were plotted for all specimens. From these graphs, the following quantities were calculated for each specimen in absolute values: (a) stiffness ( $\mathrm{N} / \mathrm{mm}$ ); (b) load to failure $(\mathrm{N})$; and (c) energy to failure $(\mathrm{mJ})$. In order to standardise outcome values, it was also considered necessary to calculate comparative values in percentage between the treatment and control groups.

All specimens, including those that were subjected to biomechanical analysis, were assessed histologically. The harvested tissue was immersed in $4 \%$ formalin solution. After 24 hours, the specimens were decalcified in a Surgipath 

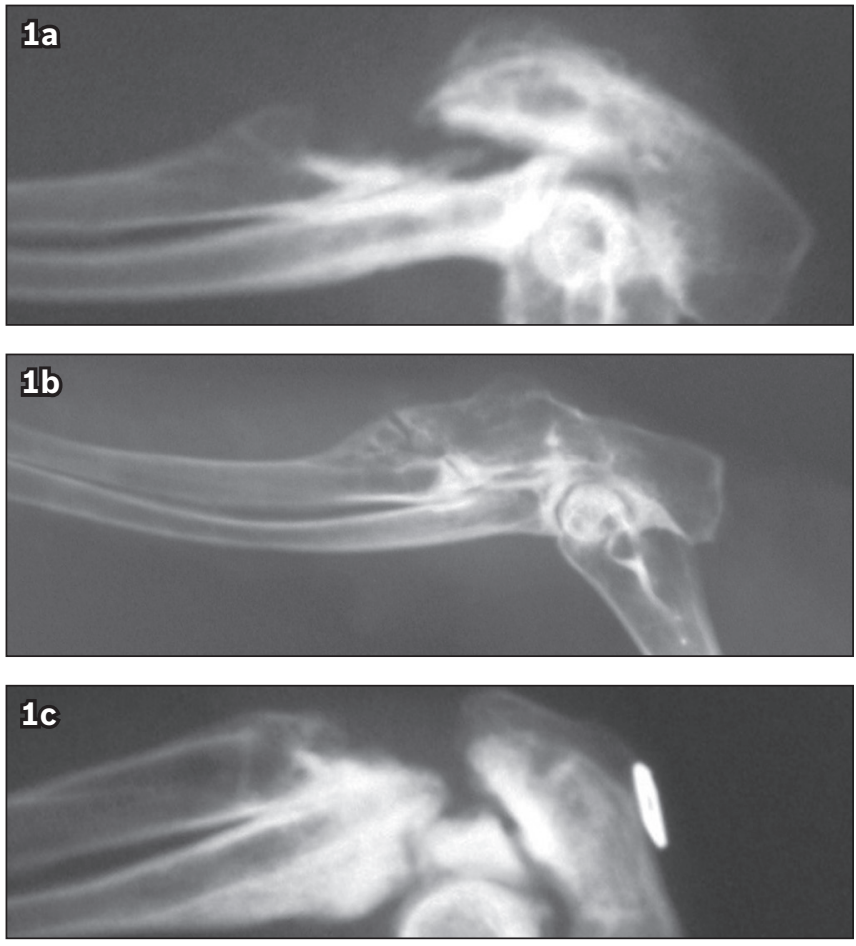

Fig. 1 Radiographs show (a) no union in Group A (platelet-rich plasma [PRP]); (b) union in Group B (PRP and demineralised bone matrix); and (c) no union in Group C (control) at postoperative week 12.

Decalcifier IIß solution (Leica Biosystems, Buffalo Grove, IL, USA). Complete decalcification was achieved in 8-10 days. The samples were then sustained via the typical histopathologic process and sectioned to a thickness of $5 \mu \mathrm{m}$. Mounted histological sections were stained using haematoxylin and eosin as well as Alizarin Red. ${ }^{(15,16)}$ Histological analysis was performed on longitudinal sections that included the site of the bone-defect interface, using the three most central sections from each specimen. A 14-point histological grading system described by Salkeld et al was used for histological grading of the healed sites. ${ }^{(11)}$ The quality of union (range 0-4), cortex development and remodelling (0-4), and the degree of bone graft incorporation and new bone formation (0-6) were assessed according to Salkeld et al's grading scale. Histological scores ranged from 0 (minimum) to 14 (maximum) and were the sum of the abovementioned parameters.

All results were expressed as mean \pm standard deviation (SD). Significant differences among groups were evaluated using chi-square test. SPSS version 11.5.0 (SPSS Inc, Chicago, IL, USA) was used for analysis and a p-value $<0.05$ indicated statistical significance.

\section{RESULTS}

Serious complications and death were not observed during our study. All the rabbits were able to walk 2-3 days postoperatively. Radiography performed immediately after surgery (Figs. 1a-c) showed segmental, critical-size defects of uniform size in the area between the upper and middle third of the ulna. Platelet concentration (mean \pm SD) of blood and PRP was $480 \pm 208 \times 10^{3} / \mu \mathrm{l}$ and 3,850 $\pm 818 \times 10^{3} / \mu \mathrm{l}$, respectively. In blood and PRP, the concentration of TGFB1 was $51.25 \pm 12.37 \mathrm{ng} / \mathrm{mL}$
Table I. Radiographic grading of degree of healing of the osseous defects, according to the scale by Cook et al. ${ }^{(12)}$

\begin{tabular}{lccc}
\hline Group & \multicolumn{3}{c}{ Mean \pm standard deviation } \\
\cline { 2 - 4 } & Immediate & $\mathbf{4}$ wk & $\mathbf{1 2}$ wk \\
\hline A & $0.70 \pm 0.50$ & $0.75 \pm 0.45$ & $0.75 \pm 0.45$ \\
B & $0.70 \pm 0.50$ & $3.55 \pm 0.79$ & $4.92 \pm 0.79$ \\
C & 0 & $0.50 \pm 0.52$ & $0.5 \pm 0.52$ \\
\hline
\end{tabular}

Scores range from 0 (no change) to 6 (defects absolutely bridged with new bone). Group A: platelet-rich plasma (PRP); Group B: PRP and demineralised bone matrix; Group C: control

Table II. Comparison of postoperative radiological scores among the three groups.

\begin{tabular}{llc}
\hline Group & \multicolumn{2}{c}{$\mathbf{p - v a l u e}$} \\
\cline { 2 - 3 } & $\mathbf{4} \mathbf{w k}$ & $\mathbf{1 2} \mathbf{~ w k}$ \\
\hline A vs. B & 0.045 & 0.029 \\
A vs. C & 0.546 & 0.572 \\
B vs. C & 0.022 & 0.019 \\
\hline
\end{tabular}

Chi-square test used to compare between groups. $p<0.05$ was considered statistically significant. Group A: platelet-rich plasma (PRP); Group B: PRP and demineralised bone matrix; Group C: control

Table III. Results of biomechanical analysis of the treated (PRP and demineralised bone matrix) and intact contralateral limbs.

\begin{tabular}{lccc}
\hline Limb & Stiffness $(\mathbf{N} / \mathbf{m m})$ & $\begin{array}{c}\text { Load to } \\
\text { failure }(\mathbf{N})\end{array}$ & $\begin{array}{c}\text { Energy to } \\
\text { failure }(\mathbf{m J})\end{array}$ \\
\hline Treated & $180.5 \pm 78.7$ & $242.5 \pm 73.8$ & $275.5 \pm 72.5$ \\
Intact & $128.6 \pm 6.7$ & $185.0 \pm 65.4$ & $180.8 \pm 51.3$ \\
\hline
\end{tabular}

Data presented as mean \pm standard deviation. PRP: platelet-rich plasma

and $195.78 \pm 40.28 \mathrm{ng} / \mathrm{mL}$, respectively, while the concentration of PDGF-BB was $3.61 \pm 0.42 \mathrm{~g} / \mathrm{mL}$ and $18.90 \pm 7.45 \mathrm{ng} / \mathrm{mL}$, respectively.

The defects filled by new bone were evaluated and the mean radiographic scores are listed in Table I. Group B (PRP and DBM) had significantly higher mean radiographic scores than Group A (PRP only) and Group C (control) at postoperative weeks 4 and 12. Defects treated with a mixture of PRP and DBM showed callus formation of significant size with a diffuse irregular appearance at 12 weeks postoperatively (Fig. 1b). New bone formation was noticed within the osseous defects, expanding into the surrounding soft tissues. Most of the defects had a radiographic score $>4$. In four of the skeletal defects in Group B, a radiolucent line across the site of the previous osteotomy could still be seen 12 weeks postoperatively although bone union was achieved (Fig. 1b).

Critical-size defects treated with PRP only (Group A) or not treated (Group C) showed minimal or no formation of new bone at postoperative weeks 4 and 12. Both Groups A and C had a mean radiographic score of 0 or 1 at 12 weeks postoperatively (Figs. 1a \& c, respectively). Statistical analysis at postoperative weeks 4 and 12 using chi-square test showed a significant difference among the three groups (Table II). A gross assessment of the specimens revealed no union in Groups $A$ and $C$, but macroscopic bone union in Group B (Fig. 2). Specimens from Groups A and C were not mechanically tested due to instability on manual palpation. The results of biomechanical analysis of the specimens in Group B are summarised in Table III. 
Table IV. Results of histological assessment.

\begin{tabular}{|c|c|c|c|}
\hline Parameter & Group A & Group B & Group C \\
\hline Quality of union $(0-4)$ & $0.920 \pm 0.289$ & $3.580 \pm 0.515$ & $0.170 \pm 0.389$ \\
\hline Cortex development and remodelling (0-4) & $0.250 \pm 0.452$ & $3.420 \pm 0.515$ & $0.170 \pm 0.389$ \\
\hline Bone graft incorporation and new bone formation $(0-6)$ & $0.420 \pm 0.515$ & $4.670 \pm 0.888$ & $0.170 \pm 0.389$ \\
\hline Total $(0-14)$ & $1.580 \pm 0.904$ & $11.670 \pm 1.497$ & $0.500 \pm 1.000$ \\
\hline
\end{tabular}

Data presented as mean \pm standard deviation. Group A: platelet-rich plasma (PRP); Group B: PRP and demineralised bone matrix; Group C: control

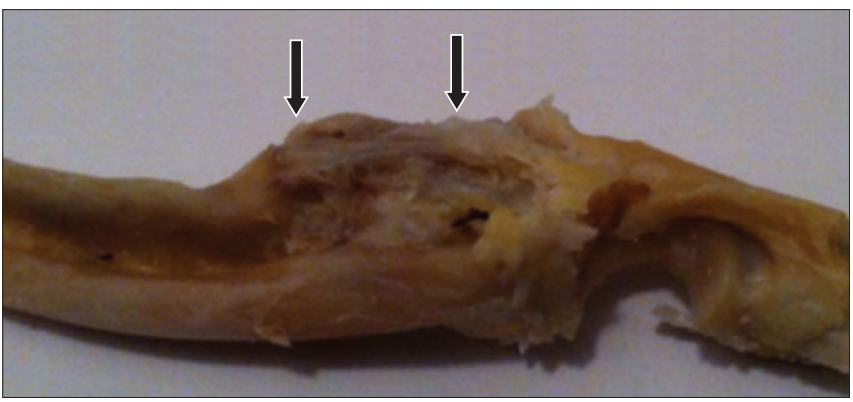

Fig. 2 Group B: photograph shows macroscopic union with significant callus (arrows) in a defect.

Table V. Comparison of histological scores among the three groups 12 weeks postoperatively.

\begin{tabular}{lc}
\hline Group & p-value \\
\hline A vs. B & 0.045 \\
A vs. C & 0.229 \\
B vs. C & 0.021 \\
\hline
\end{tabular}

Chi-square test used to compare between groups. $\mathrm{p}<0.05$ was considered statistically significant. Group A: platelet-rich plasma (PRP); Group B: PRP and demineralised bone matrix; Group C: control

Comparative values in percentage were also calculated between the treated and intact contralateral specimens of Group B. The treated specimens had average stiffness, load to failure and energy to failure values that were $88.0 \%, 153.0 \%$ and $152.5 \%$ more than the intact contralateral specimens of Group B, respectively. Histological scores for the three groups are shown in Table IV.

The defects filled with PRP only and those that were not treated showed minimal or no formation of new bone. Histologically, these defects also had the most fibrous tissue bridging them. Defects treated with PRP and DBM, however, were bridged with new bone formation. The newly formed bone was found inside the defect and also extended into the surrounding soft tissue, creating an oversized callus at 12 weeks postoperatively. There were mineralised areas of cartilage, new osteoid deposition and complete bone formation with mature bone, as evidenced by the presence of lamellar bone deposition and medullary cavities (Figs. 3a \& b). Similar to the radiological results, statistical analysis with chi-square test showed a significant difference between the histological scores of the three groups (Table V).

The histological scores for osseous defects treated with platelet-rich plasma and demineralised bone matrix were significantly greater than that of the other groups; Groups A and $C$ achieved scores of about 0 or $1(p<0.05)$. Inside the callus, there was complete absorption of the graft as new bone was
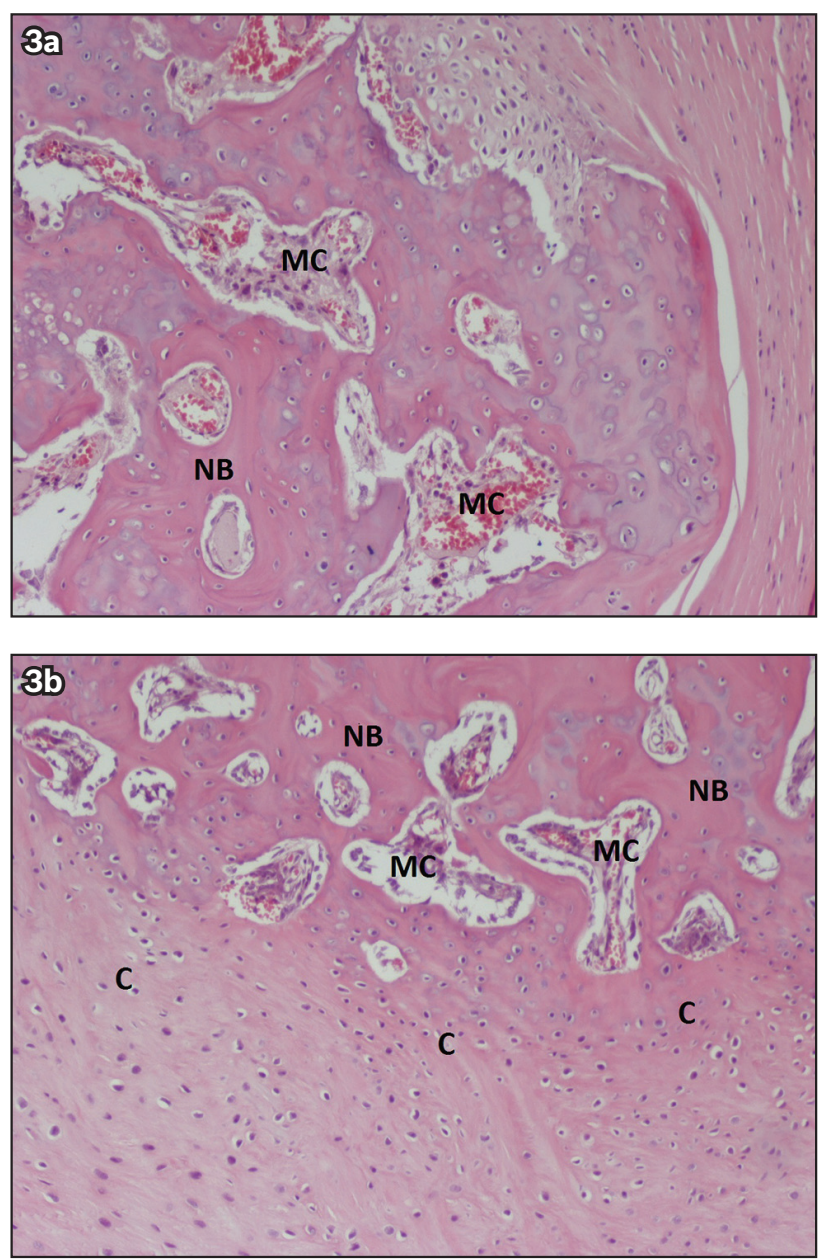

Fig. 3 ( $a$ \& b) Photomicrographs from Group B show new bone formation in undecalcified histological sections of a critical-size defect treated with platelet-rich plasma and demineralised bone matrix. There is endochondral ossification (bone margin of the defect), presence of medullary cavities, xenograft resorption and filling of the defect with new bone (Haematoxylin \& eosin, $\times 200)$. C: cartilage; MC: medullary cavities; NB: new bone

produced, thus showing that new bone replaced the xenograft. All the groups did not show complete development of cortices 12 weeks after the operation, although specimens from Group B had partial cortex formation and recreation of marrow space.

\section{DISCUSSION}

Autologous PRP is prepared from blood after simple centrifugation to concentrate platelets. Platelets contain alpha granules that are rich in several chemotactic and proliferative growth factors, such as the PDGF, TGFB1, insulin-like growth factor, vascular endothelial growth factor, epidermal growth factor and many others that play key roles in tissue repair mechanisms and bone healing. ${ }^{(17,18)}$ 
Upon application to the fracture site and activation with thrombin or calcium chloride solution, platelets contained in PRP release many of these growth factors and cytokines. They may induce the healing of tissue at the cellular level, as they influence cells involved in tissue regeneration. They also can stimulate the formation of blood vessels at the fracture site (angiogenesis) and migration of mesenchymal stem cells, monocytes and macrophages. Additionally, these platelets can promote cellular differentiation and further aggregation of platelets. ${ }^{(19,20)}$ Thus, these growth factors do not directly stimulate bone formation but affect the above critical repair functions. ${ }^{(3)}$

DBM preparations contain osteoconductive materials with some osteoinductive properties. ${ }^{(2)}$ Ideally, the combination of PRP and DBM induces bone regeneration. A few studies have reported that when applied to osseous defects, PRP with DBM induces bone healing, (21) while other studies concluded that PRP with DBM inhibits fracture healing. ${ }^{(7,22)}$ However, these latter studies have some critical differences from the present study; they either used different species of animals, with different metabolisms, or had a smaller sample size. Also, these studies applied the mixture of PRP and DBM to different sites, leading to varying levels of DBM activity and subsequently affecting osteogenic ability and the course of bone formation. ${ }^{(8,23)}$ Lastly, different PRP preparation devices were used.

The results of this study suggest that PRP combined with DBM may enhance bone healing of segmental defects, while application of PRP alone does not lead to bone union. The human DBM (xenograft) acted as a scaffold with osteoconductive properties; this was combined with the osteoinductive properties of autologous PRP. Significant enhancement of bone union was found in these critical defects 12 weeks postoperatively. The new bone filled the defects and expanded to the surrounding soft tissue. The callus that formed appeared to show cortex formation and remodelling of new bone, indicating incorporation of graft with new bone. Biomechanical and histological findings only showed enhancement of bone healing in Group B specimens.

This study used PRP in a gel form (activated with calcium chloride solution) alone or with DBM for better anchorage in the bone defects. New bone formation and filling of defects was achieved with the use of PRP combined with DBM. Some studies have concluded that PRP can increase the osteoinductivity of DBM when used without thrombin activation, as thrombin activation may lead to faster release of growth factors in PRP to the application site. ${ }^{(8)}$ The use of xenogenous DBM was not a limitation of this study because it does not have any significant osteoinductive properties, and hence represented a purely osteoconductive material when mixed with osteoinductive PRP to fill the defects. In the present study, PRP was prepared according to the manufacturer's instructions and had 3-7 times more platelets and growth factors. Bone healing may not be improved by using PRP with very high platelet concentrations that was obtained via aggressive processing techniques. ${ }^{(24,25)}$ The preparation and use of PRP depend on many variables such as the centrifugation method and delivery system. ${ }^{(26)}$
The rabbit is an appropriate model for PRP experimental studies, as its haematological status is similar to that of humans. Rabbits have an average platelet count of $400,000 / \mathrm{mm}^{3}$. They also have a specific sequence of phases for bone healing that lasts for about 2-3 months after the operation. However, a limitation of this study could be the different (faster) rhythm of bone healing in rabbits compared to humans. ${ }^{(27-29)}$ Another notable fact was that postoperative external immobilisation was not used in the rabbit forearm with the defect. The integrity of the radius and the strong fibro-osseous radioulnar union proximal and distal to the skeletal defect provided mechanical stability. ${ }^{(11)}$ In addition, external immobilisation of a rabbit forearm would be difficult.

PRP has previously been applied alone or in combination with autografts or other bone grafts to enhance bone union. According to the literature, the use of PRP for osseous healing appears beneficial only when it is mixed with osteoconductive scaffolds. ${ }^{(25)}$ The results of the present study support these findings. Although DBM has shown some osteoinductive activity in animal studies, it has never demonstrated this effect in clinical use. ${ }^{(3,9)}$ This may be a possible limitation of the study, and future studies should address this question by including a group in which only DBM is used to fill bone defects.

Few studies in the literature have used large skeletal defects of this size filled with a material other than autograft, or osseous defects filled with autologous PRP (alone or combined with DBM). Although the primary aim of the present study was to show the effectiveness of PRP for bone enhancement, and not to compare it with the well-established autograft or DBM alone, the exclusion of the autograft or DBM groups may have been another limitation of this study, and these groups should be included in future research.

Additionally, different DBM products may vary in their osteogenic properties in animal studies. Most studies revealed no effect of PRP on bone healing, while a small number showed enhancement of bone healing. ${ }^{(6)}$ Research involving a larger number of animals may be needed to support the encouraging results of PRP and enhancement of bone union found in our study.

PRP has been widely used in orthopaedic, periodontal and maxillofacial surgery with mixed results. It may initiate the process of fracture healing, acting during the early stages of bone healing. ${ }^{(30)}$ Thus, transferring an increased concentration of platelets as PRP to a fracture site is advisable, provided that the preparation and application of PRP are not overly challenging in clinical practice, and may benefit bone and soft tissue healing with no side effects. Better understanding and knowledge of the mechanisms of PRP are required. The potential efficacy of PRP is controversial and more research is necessary.

In conclusion, this study found that enhancement of bone healing in critical-size defects was statistically significant when PRP was combined with DBM, while bridging of osseous defects with PRP alone did not seem to have any positive effect compared to the control group. Enhancement of bone union was shown in radiological findings, gross assessment, and biomechanical and histological results. Therefore, PRP was found to be effective only when used in combination with a bone graft. 


\section{ACKNOWLEDGEMENTS}

The authors would like to thank the Hellenic Association of Orthopaedic Surgery and Traumatology for its support and contribution to our study.

\section{REFERENCES}

1. Younger EM, Chapman MW. Morbidity at bone graft donor sites. J Orthop Trauma 1989; 3:192-5.

2. Lieberman JR, Daluiski A, Einhorn TA. The role of growth factors in the repair of bone. Biology and clinical applications. J Bone Joint Surg Am 2002; 84-A:1032-44.

3. De Long WG Jr, Einhorn TA, Koval K, et al. Bone grafts and bone graft substitutes in orthopaedic trauma surgery. A critical analysis. J Bone Joint Surg Am 2007 89:649-58.

4. Kazakos K, Lyras DN, Verettas D, Tilkeridis K, Tryfonidis M. The use o autologous PRP gel as an aid in the management of acute trauma wounds. Injury 2009; 40:801-5.

5. Gosens T, Peerbooms JC, van Laar W, den Oudsten BL. Ongoing positive effect of platelet-rich plasma versus corticosteroid injection in lateral epicondylitis: a double-blind randomized controlled trial with 2-year follow-up. Am J Sports Med 2011; 39:1200-8

6. Ahmad Z, Howard D, Brooks RA, et al. The role of platelet rich plasma in musculoskeletal science. JRSM Short Rep 2012; 3:40.

7. Faratzis G, Leventis M, Chrysomali E, et al. Effect of autologous platelet-rich plasma in combination with a biphasic synthetic graft material on bone healing in critical-size cranial defects. J Craniofac Surg 2012; 23:1318-23.

8. Han B, Woodell-May J, Ponticiello M, Yang Z, Nimni M. The effect of thrombin activation of platelet-rich plasma on demineralized bone matrix osteoinductivity. J Bone Joint Surg Am 2009; 91:1459-70.

9. Bolander ME, Balian G. The use of demineralized bone matrix in the repair of segmental defects. Augmentation with extracted matrix proteins and a comparison with autologous grafts. J Bone Joint Surg Am 1986; 68:1264-74.

10. Heiple KG, Chase SW, Herndon CH. A comparative study of the healing process following different types of bone transplantation. J Bone Joint Surg Am 1963; 45:1593-616

11. Salkeld SL, Patron LP, Barrack RL, Cook SD. The effect of osteogenic protein-1 on the healing of segmental bone defects treated with autograft or allograft bone. J Bone Joint Surg Am 2001; 83-A:803-16.

12. Cook SD, Wolfe MW, Salkeld SL, Rueger DC. Effect of recombinant human osteogenic protein-1 on healing of segmental defects in non-human primates. J Bone Joint Surg Am 1995; 77:734-50.

13. Karachalios T, Boursinos L, Poultsides L, Khaldi L, Malizos KN. The effects of the short-term administration of low therapeutic doses of anti-COX-2 agents on the healing of fractures. An experimental study in rabbits. J Bone Joint Surg Br 2007; 89:1253-60.

14. Turner $\mathrm{CH}$, Burr DB. Basic biomechanical measurements of bone: a tutorial. Bone 1993; 14:595-608.

15. Hood RC, Neill WM. A modification of alizarin red S technic for demonstrating bone formation. Stain Technol 1948; 23:209-18.

16. Yoshiki S. A simple histological method for identification of osteoid matrix in decalcified bone. Stain Technol 1973; 48:233-8.

17. Bostrom MP. Expression of bone morphogenetic proteins in fracture healing. Clin Orthop Relat Res 1998; 355 Suppl: S116-23.

18. Bouxsein ML, Turek TJ, Blake CA, et al. Recombinant human bone morphogenetic protein-2 accelerates healing in a rabbit ulnar osteotomy model. J Bone Joint Surg Am 2001; 83-A:1219-30.

19. Slater M, Patava J, Kingham K, Mason RS. Involvement of platelets in stimulating osteogenic activity. J Orthop Res 1995; 13:655-63.

20. Marx RE, Carlson ER, Eichstaedt RM, et al. Platelet-rich plasma: Growth factor enhancement for bone grafts. Oral Surg Oral Med Oral Pathol Oral Radiol Endod 1998; 85:638-46.

21. Clokie CM, Moghadam H, Jackson MT, Sandor GK. Closure of critical sized defects with allogenic and alloplastic bone substitutes. J Craniofac Surg 2002; 13:111-21.

22. Ranly DM, McMillan J., Keller T, et al. Platelet-derived growth factor inhibits demineralized bone matrix-induced intramuscular cartilage and bone formation. A study of immunocompromised mice. J Bone Joint Surg Am 2005; 87:2052-64.

23. Urist MR. The search for and discovery of bone morphogenetic proteins (BMP). In: Urist MR, O'Connor BT, Burwell RG, eds. Bone grafts, derivatives and substitutes. London: Butterworth-Heinemann, 1994: 315-62.

24. Woodell-May JE, Ridderman DN, Swift MJ, Higgins J. Producing accurate platelet counts for platelet rich plasma: validation of a hematology analyzer and preparation techniques for counting. J Craniofac Surg 2005; 16:749-56.

25. Malhotra A, Pelletier MH, Yu Y, Walsh WR. Can platelet-rich plasma (PRP) improve bone healing? A comparison between the theory and experimental outcomes. Arch Orthop Trauma Surg 2013; 133:153-65.

26. Lin SS, Landesberg R, Chin HS, Lin J, Eisig SB, Lu HH. Controlled release of PRP-derived growth factors promotes osteogenic differentiation of human mesenchymal stem cells. Conf Proc IEEE Eng Med Biol Soc 2006; 1:4358-61.

27. Butterfield KJ, Bennett J, Gronowicz G, Adams D. Effect of platelet-rich plasma with autogenous bone graft for maxillary sinus augmentation in a rabbit model. J Oral Maxillofac Surg 2005; 63:370-6.

28. Watanabe K, Niimi A, Ueda M. Autogenous bone grafts in the rabbit maxillary sinus. Oral Surg Oral Med Oral Pathol Oral Radiol Endod 1999; 88:26-32.

29. Albrektsson T, Linder L. Intravital, long-term follow-up of autologous experimental bone grafts. Arch Orthop Trauma Surg 1981; 98:189-93.

30. Dhillon RS, Schwarz EM, Maloney MD. Platelet-rich plasma therapy - future or trend? Arthritis Res Ther 2012; 14:219. 